\title{
Diagnostics of a charge breeder electron cyclotron resonance ion source helium plasma with the injection of ${ }^{23} \mathrm{Na}^{1+}$ ions
}

\author{
O. Tarvainen ${ }^{*}$ and H. Koivisto \\ Department of Physics, University of Jyväskylä, 40500 Jyväskylä, Finland \\ A. Galatà \\ INFN-Laboratori Nazionali di Legnaro, Viale dell'Università 2, 35020 Legnaro, Padova, Italy \\ J. Angot, T. Lamy, and T. Thuillier \\ LPSC, Université Grenoble-Alpes, CNRS/IN2P3, 53 avenue des Martyrs, 38026 Grenoble Cedex, France \\ P. Delahaye and L. Maunoury \\ GANIL, CEA/DSM-CNRS/IN2P3, 14076 Caen Cedex 05, France \\ D. Mascali and L. Neri \\ INFN-Laboratori Nazionali del Sud, Via S. Sofia 62, 95123 Catania, Italy
}

(Received 16 March 2016; published 16 May 2016)

\begin{abstract}
This work describes the utilization of an injected ${ }^{23} \mathrm{Na}^{1+}$ ion beam as a diagnostics of the helium plasma of a charge breeder electron cyclotron resonance ion source. The obtained data allows estimating the upper limit for the ion-ion collision mean-free path of the incident sodium ions, the lower limit of ion-ion collision frequencies for all charge states of the sodium ions and the lower limit of the helium plasma density. The ion-ion collision frequencies of high charge state ions are shown to be at least on the order of $1-10 \mathrm{MHz}$ and the plasma density is estimated to be on the order of $10^{11} \mathrm{~cm}^{-3}$ or higher. The experimental results are compared to simulations of the ${ }^{23} \mathrm{Na}^{1+}$ capture into the helium plasma. The results indicate that the lower breeding efficiency of light ions in comparison to heavier elements is probably due to different capture efficiencies in which the in-flight ionization of the incident $1+$ ions plays a vital role.
\end{abstract}

DOI: 10.1103/PhysRevAccelBeams.19.053402

\section{INTRODUCTION}

Charge breeder ion sources are essential postaccelerator components in radioactive ion beam facilities allowing the study of e.g. the properties of exotic nuclei [1]. Two types of plasma ion sources, namely electron cyclotron resonance ion sources (ECRIS) and electron beam ion sources have been successfully employed for the purpose [2,3]. Although both methods have their benefits and drawbacks [4,5], their fundamental principle is similar; low charge state ions (typically $1+$ ) are injected into the charge breeder, their charge state is increased by electron impact ionization and, finally, highly charged ions are extracted and postaccelerated.

In an ECRIS charge breeder the charge multiplication occurs in the plasma heated by microwave radiation and confined by a minimum-B magnetic field. It has been recently demonstrated [6] that the $1+$ injection can be

*olli.tarvainen@jyu.fi

Published by the American Physical Society under the terms of the Creative Commons Attribution 3.0 License. Further distribution of this work must maintain attribution to the author $(s)$ and the published article's title, journal citation, and DOI. applied for diagnostics of the ECRIS plasma parameters. The method is based on the analysis of the transmission of the uncaptured fraction of the incident $1+$ ion beam and yields information on the mean-free path of the injected ions, ion-ion collision frequencies and plasma (electron) density. The experiments described in Ref. [6] were conducted with ${ }^{85} \mathrm{Rb}^{1+}$ and ${ }^{133} \mathrm{Cs}^{1+}$ ions injected into an oxygen plasma. Here we describe experimental results obtained with ${ }^{23} \mathrm{Na}^{1+}$ ions injected into a helium plasma. The work demonstrates the applicability of the diagnostics method for a combination of lighter ions. Since the ionization cross section for $\mathrm{Na}^{1+}$ ions is approximately an order of magnitude smaller than the corresponding cross section for the heavier alkali metals [7], the uncertainties of the diagnostics results related to in-flight ionization are reduced. Moreover, the results can be used to identify the main reason for the charge breeding efficiency of low mass species, e.g. ${ }^{23} \mathrm{Na}$, being typically poor in comparison to heavier ions, e.g. ${ }^{85} \mathrm{Rb}$.

\section{EXPERIMENTAL SETUP FOR ${ }^{23} \mathrm{Na}^{1+} \rightarrow{ }^{23} \mathrm{Na}^{n+}$ CHARGE BREEDING}

The experimental data were taken with the $14.5 \mathrm{GHz}$ PHOENIX ECR charge breeder [8] at Laboratoire de 
Physique Subatomique et de Cosmologie (LPSC). The ${ }^{23} \mathrm{Na}^{1+}$ ion beams were produced with a thermionic ion gun by Heat Wave Labs. The energy of the $1+$ ions injected into the charge breeder was controlled experimentally by adjusting the voltage difference $\Delta V$ between the $1+$ ion source and the ECRIS charge breeder. Due to a positive plasma potential, $\Delta V$ must be negative in order to prevent reflection of the incident $1+$ ions. The charge bred ${ }^{23} \mathrm{Na}^{n+}$ ions are extracted from the ECRIS together with the buffer gas ions and $m / q$-analyzed with a bending magnet. The charge breeding efficiencies were determined by measuring the intensity of the incident $1+$ ion beam upstream from the charge breeder ECRIS and the intensities of the extracted $m / q$-analyzed $n+$ beams at the end of the beam line. The $n+$ currents were also measured after switching off the $1+$ injection and the remaining "background current" was subtracted when calculating the efficiencies for different charge states. The charge breeding times were measured by pulsing the injected $1+$ ion beam with electrostatic deflector plates located immediately downstream from the $1+$ ion source and recording the corresponding $n+$ Faraday cup signals. All beam current measurements were performed with a picoammeter (Keithley 6485), which has an accuracy of $0.4 \%$ in the relevant intensity range. A schematic presentation of the experimental setup in its present form can be found e.g. from [6].

All experiments described hereafter were performed using helium as a buffer gas for the charge breeder ECR discharge. It has been observed experimentally that helium is superior, in comparison to oxygen for example, for optimizing the breeding efficiencies of high charge state $\mathrm{Na}$ and $\mathrm{K}$ ions [9]. This is believed to be due to the so-called gas mixing effect [10] i.e. the increase of the extracted currents of highly charged ions of a heavier element by mixing lighter gas into the ECR discharge. Since the discovery of the technique, several mechanisms have been proposed to explain the effect $[10,11]$. In the case of the charge breeder, the most probable cause is ion cooling $[12,13]$ resulting from the mass effect in ion-ion collisions, that makes $\mathrm{Na}-\mathrm{He}$ collisions more effective than $\mathrm{Na}-\mathrm{O}$ ones. In this respect the behavior of the charge breeder is similar to conventional ECRISs in which helium is often used as a buffer gas to optimize the extracted currents of ions up to mass number $A=30$ (approximately).

\section{DESCRIPTION OF THE DIAGNOSTICS METHOD}

The $1+$ ion beam injected into the charge breeder ECRIS can be used for plasma diagnostics. This is based on the fact that there are two populations in the $m / q$-spectrum of the extracted ion beams: the low $(1+$ and $2+)$ charge states, representing the uncaptured fraction of the incident $1+$ ion beam, and the high charge states, that have been captured in ion-ion collisions and subsequently charge bred through electron impact ionization [6,14]. Measurement of the
$1+$ ion beam propagating through the charge breeder plasma allows estimating the lower limits of ion-ion collision frequency and plasma density in the ECRIS plasma, as summarized below and thoroughly described in Ref. [6].

It is widely accepted that the thermalization and capture of the incident $1+$ beam is optimized when the longitudinal component of the incident ion velocity $v_{a}$ equals the most probable velocity of the plasma ions $v_{b}$ [15]. However, recent simulations of rubidium charge breeding in oxygen plasma $[16,17]$ have revealed that an extra energy is necessary to allow the injected ions to propagate and become stopped deep inside the dense plasma, i.e.

$$
v_{a} \geq v_{b}=\sqrt{\frac{2 k T_{b}}{m_{b}}} .
$$

In practice, the energy of the incident $1+$ ions is controlled by adjusting the voltage difference $\Delta V$ between the $1+$ ion source and the charge breeder ECRIS (plasma). The $\Delta V$ dependence of low and high charge state ion beams extracted from the charge breeder is significantly different, as demonstrated in Fig. 1(a) comparing the normalized breeding efficiencies of ${ }^{23} \mathrm{Na}^{1+}$ and ${ }^{23} \mathrm{Na}^{6+}$ as a function of $\Delta V$ (negative): the efficiencies of high charge states (e.g. $6+$ ) are nonzero only when $5 \mathrm{~V} \leq \Delta V \leq 25 \mathrm{~V}$, while the extracted current of $1+$ increases monotonically with $\Delta V$. The result is consistent with the interpretation of the $1+$ ions representing the fraction of the uncaptured incident ions propagating through the plasma and the high charge states representing the ion population that has been successfully captured and ionized. Further support for this interpretation is provided in Fig. 1(b) showing the transit time of the $\mathrm{Na}^{1+}$ ions through the charge breeder with and without igniting the ECR discharge. The temporal characteristics of the two curves are virtually identical, which implies that the $1+$ beam extracted from the charge breeder represents a fraction of the incident beam being transported through the helium plasma.

Identifying the two populations in the $m / q$-spectrum of ion beams extracted from the charge breeder allows estimating the capture and ionization efficiencies. Furthermore, the data can be used for estimating the mean-free path of cumulative long range ion-ion collisions of the $\mathrm{Na}^{1+}$ ions. The mean-free path, $\lambda_{i i}^{1+}$, can be calculated from

$$
\lambda_{i i}^{1+}=-\frac{L}{\frac{L}{\lambda_{i}}+\ln \left(\frac{I_{p}}{I_{v}}\right)},
$$

where $L$ is the effective plasma length, namely the estimated length of the ion trajectories in the dense plasma inside the ECR zone, $\lambda_{i}$ the mean-free path of in-flight electron impact ionization $\left(\mathrm{Na}^{1+}+e \rightarrow \mathrm{Na}^{2+}+2 e\right), I_{p}$ the transported fraction of the injected $1+$ beam with 

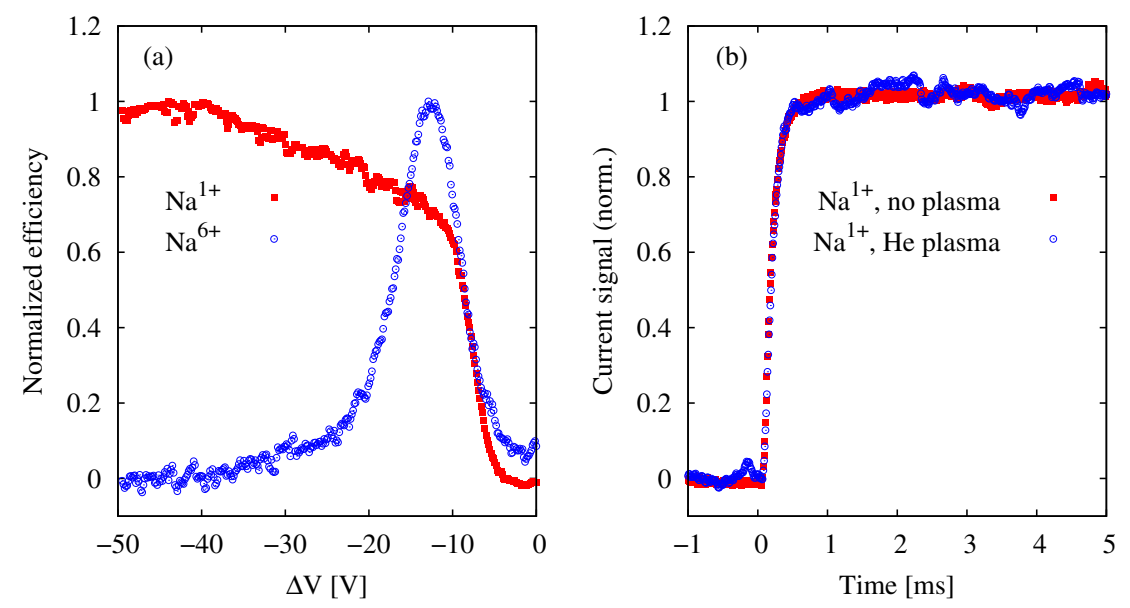

FIG. 1. (a) Normalized breeding efficiencies of ${ }^{23} \mathrm{Na}^{1+}$ and ${ }^{23} \mathrm{Na}^{6+}$ as a function of $\Delta V$. (b) Time-resolved Faraday cup signals of ${ }^{23} \mathrm{Na}^{1+}$ with and without helium plasma in the charge breeder $(\Delta V=-12.9 \mathrm{~V})$.

charge breeder plasma and $I_{v}$ the corresponding fraction in vacuum i.e. without igniting the plasma [6]. The corresponding collision frequency of the $1+$ ions can be calculated from the mean-free path by assuming that the ions propagate through the plasma with a given initial velocity $v_{a}$ until they experience an elastic collision with the plasma ions, i.e.

$$
\nu_{i i}=\frac{v_{a}}{\lambda_{i i}}
$$

Because the Rutherford scattering cross section in ion-ion collisions, $\sigma$, is proportional to the square of the ion charge (state), $q$, i.e. $\sigma \propto q^{2}$, the ion-ion collision frequency also scales as $\nu_{i i}^{q+} \propto q^{2}$. The scaling allows one to estimate the upper limit of the ion mean-free paths and lower limit of the ion-ion collision frequency for different charge states of $\mathrm{Na}$ ions (as explained in detail in Ref. [6]). On the other hand the cumulative large angle ion-ion collision frequency [18] can be expressed as

$$
\nu_{i j}=\frac{6.8 \times 10^{-8} \ln \Lambda}{T_{i}^{3 / 2}} \frac{q_{i}^{2}}{A_{i}} \sum_{j} \sqrt{A_{r}} \sum_{q} n_{j}^{q} q_{j}^{2},
$$

where $A_{i}$ is the mass number of ion species $i(\mathrm{Na})$,

$$
A_{r}=\sqrt{A_{i} A_{j} /\left(A_{i}+A_{j}\right)}
$$

the reduced mass in center of mass coordinate system with $A_{j}$ being the mass number of the secondary (buffer) gas species (He) and $\sum_{q} n_{j}^{q} q_{j}^{2}$ can be expressed with the plasma (electron) density, $n_{e}$, and the average (effective) charge state of the breeder plasma ions, $q_{\mathrm{eff}}$, as

$$
\sum_{q} n_{j}^{q} q_{j}^{2}=q_{\mathrm{eff}} n_{e}
$$

In this formulation [18] the ion temperature $T_{i}$ is given in units of $\mathrm{eV}$ and electron density $n_{e}$ in units of $\mathrm{cm}^{-3}$. Mutual collisions between alkali ions can be neglected due to the fact that their feed rate is on the order of 0.1-1 $\mathrm{p} \mu \mathrm{A}$ while typical helium feed rates are approximately 3 orders of magnitude higher. Taking into account the ionization degree of the injected helium gas being several tens of percent it can be assumed that the density of the $\mathrm{Na}$ ions is insignificant in comparison to He ions. Making reasonable assumptions about the ion temperature and effective charge of the plasma allows one to estimate the order of magnitude for the plasma (electron) density as described in the following section. Typical uncertainties related to the diagnostics results are described in Ref. [6].

\section{EXPERIMENTAL RESULTS}

The breeding efficiencies of low and high charge state $\mathrm{Na}$ ions were studied as a function of the $14.5 \mathrm{GHz}$ microwave power used for sustaining the helium discharge. The measured efficiencies of low and high charge states are presented in Table I. The values were obtained at $\Delta V$ corresponding to optimized breeding efficiency of the high charge state ions with the injected ${ }^{23} \mathrm{Na}^{1+}$ beam current of 544-560 nA. Let us note that the given variation of the $1+$ beam intensity has no influence on the measured charge breeding efficiencies. It is evident from Table I that a significant fraction of the injected ions propagate through the plasma without becoming thermalized. For $470 \mathrm{~W}$ of microwave power the global charge breeding efficiency, including all $\mathrm{Na}$ charge states, was measured to be $21 \%$. It should be noted that the contribution of the $1+$ charge state, mainly propagating through the plasma, was approximately half of this value.

The estimated mean-free paths and lower limits of the ion-ion collision frequencies for $\mathrm{Na}^{1+}$ ions at different microwave powers are shown in Table II. The mean-free 
TABLE I. Breeding efficiencies (percentages) of low and high charge state ${ }^{23} \mathrm{Na}$ in helium plasma as a function of the microwave power. $\Delta V$ is optimized for $\mathrm{Na}^{6+}$ at each power.

\begin{tabular}{lrrrr}
\hline \hline Power [W] & 150 & 250 & 350 & 470 \\
\hline $\mathrm{Na}^{1+}$ & 35.7 & 15.3 & 10.8 & 8.5 \\
$\mathrm{Na}^{2+}$ & 2.5 & 1.3 & 1.6 & 1.2 \\
$\mathrm{Na}^{6+}$ & 1.1 & 1.2 & 2.2 & 1.9 \\
$\mathrm{Na}^{7+}$ & 0.5 & 0.7 & 1.1 & 1.1 \\
\hline \hline
\end{tabular}

paths are calculated with Eq. (2) assuming that the effective plasma length is $15 \mathrm{~cm}$, which corresponds to the length of the ion trajectories inside the closed ECR zone. It is also assumed that the mean-free path of ionization is significantly longer than the mean-free path of ion-ion collisions, i.e. $\lambda_{i} \gg \lambda_{i i}^{1+}$. The error caused by this assumption is discussed later. The values for the $1+$ ion beam currents in the presence the plasma correspond to the efficiencies reported in Table I. The extracted $1+$ current without plasma is taken to be $80 \%$ of the injected current, corresponding to the measured transport efficiency. Furthermore, it is assumed that the energy of the injected $\mathrm{Na}$ ions reaching the dense plasma region is on the order of few $\mathrm{eV}$. In reality the thermalization of the $1+$ ions is a result of several cumulative small angle collisions [19] but, in order to deduce the lower limit of the ion-ion collision frequency, it is considered here that a single large angle scattering event is sufficient for the capture process.

The estimated upper limits of the ion mean-free paths and lower limits of the ion-ion collision frequency for different charge states of $\mathrm{Na}$ are displayed in Table III. It is assumed here that the temperature of the plasma ions (helium) is on the order of $1 \mathrm{eV}$ [20], and that the ${ }^{23} \mathrm{Na}^{1+}$ ions are injected at the optimized energy of about $6 \mathrm{eV}$ corresponding to the condition $v_{a}=v_{b}$, which translates to

$$
E_{a}=\frac{m_{a}}{m_{b}} k T_{i}
$$

The mean-free paths and collision frequencies of the charge states $\geq 2+$ are calculated from the $1+$ mean-free paths and collision frequencies using the $\propto q^{2}$ scaling as discussed above.

The given ion-ion collision frequencies of charge states $\geq 2+$ truly represent the lower limit because, first of all, it is assumed that a single collision is sufficient to capture the

TABLE II. Estimated mean-free paths and lower limits of ion-ion collision frequency of ${ }^{23} \mathrm{Na}^{1+}$ ions injected into helium plasma.

\begin{tabular}{lcccc}
\hline \hline Power $[\mathrm{W}]$ & 150 & 250 & 350 & 470 \\
\hline$\lambda_{i i}^{1+}[\mathrm{mm}]$ & 186 & 97 & 75 & 67 \\
$\nu_{i i}^{1+}[\mathrm{kHz}]$ & 35 & 67 & 86 & 96 \\
\hline \hline
\end{tabular}

TABLE III. Mean-free paths (upper limit) and ion-ion collision frequencies (lower limit) for different charge states of $\mathrm{Na}$ ions in helium plasma.

\begin{tabular}{lcccc}
\hline \hline Power [W] & 150 & 250 & 350 & 470 \\
\hline \multicolumn{4}{l}{ Mean-free path $\lambda_{i i}^{n+}}$, & upper limit $[\mathrm{mm}]$ \\
$\mathrm{Na}^{1+}$ & 186 & 97 & 75 & 67 \\
$\mathrm{Na}^{2+}$ & 47 & 24 & 19 & 17 \\
$\mathrm{Na}^{3+}$ & 21 & 11 & 8.3 & 7.4 \\
$\mathrm{Na}^{4+}$ & 12 & 6.1 & 4.7 & 4.2 \\
$\mathrm{Na}^{5+}$ & 7.4 & 3.9 & 3.0 & 2.7 \\
$\mathrm{Na}^{6+}$ & 5.2 & 2.7 & 2.1 & 1.9 \\
$\mathrm{Na}^{7+}$ & 3.8 & 2.0 & 1.5 & 1.4 \\
$\mathrm{Na}^{8+}$ & 2.9 & 1.5 & 1.2 & 1.0 \\
$\mathrm{Ion}^{-1}$ & & & & \\
$\mathrm{Na}^{1+}$ & 0.038 & 0.073 & 0.094 & 0.11 \\
$\mathrm{Na}^{2+}$ & 0.15 & 0.29 & 0.38 & 0.42 \\
$\mathrm{Na}^{3+}$ & 0.34 & 0.66 & 0.85 & 0.95 \\
$\mathrm{Na}^{4+}$ & 0.61 & 1.2 & 1.5 & 1.7 \\
$\mathrm{Na}^{5+}$ & 0.95 & 1.8 & 2.4 & 2.6 \\
$\mathrm{Na}^{6+}$ & 1.4 & 2.6 & 3.4 & 3.8 \\
$\mathrm{Na}^{7+}$ & 1.9 & 3.6 & 4.6 & 5.2 \\
$\mathrm{Na}^{8+}$ & 2.4 & 4.7 & 6.0 & 6.8 \\
\hline \hline
\end{tabular}

incident $1+$ ions whereas several cumulative large angle collisions could be necessary (as discussed above) and, second, high charge state $\mathrm{Na}$ ions are not assumed to be fully thermalized with helium plasma ions i.e. their collision frequencies are not corrected by a scaling factor taking into account the difference between the incident ion energy and plasma ion average energy. This correction would be due to the fact that the ion-ion collision frequency scales with the ion energy (or temperature) as $\nu_{i i} \propto E_{i}^{-3 / 2}$ (or $\nu_{i i} \propto T_{i}^{-3 / 2}$ [18]) hence increasing the ion-ion collision frequencies of thermalized ions by approximately an order of magnitude from the given values. Only the charge states detected in the extracted $m / q$-spectrum are listed in the table. The values listed in the table imply that all thermalized charge states of $\mathrm{Na}(\geq 3+)$ are collisional, i.e. their collision frequencies are higher than their gyrofrequencies in the dense plasma region of the ECRIS magnetic field.

In order to estimate the minimum electron density (averaged over the length of ion trajectories) we use an ion temperature of $0.5 \mathrm{eV}$ (lower limit) and value of 10 for the Coulomb logarithm (upper limit). Furthermore, it is estimated from the recorded $m / q$ spectra that the average ion charge state in the helium plasma is always less than 1.5 (upper limit) corresponding to high microwave power $(470 \mathrm{~W})$ without $1+$ injection and taking into account the impurities in the spectrum. The estimated lower limit of the plasma density as a function of the applied microwave power is presented in Table IV.

Neglecting the in-flight ionization causes an error to the calculated ion-ion collision frequencies and plasma densities as described in Ref. [6]. Using similar assumptions 
TABLE IV. Estimated lower limit for the charge breeder helium plasma (electron) density as a function of microwave power.

\begin{tabular}{lcccc}
\hline \hline Power [W] & 150 & 250 & 350 & 470 \\
\hline Plasma density $\left[\mathrm{cm}^{-3}\right]$ & $1.6 \times 10^{11}$ & $3.1 \times 10^{11}$ & $4.0 \times 10^{11}$ & $4.4 \times 10^{11}$ \\
\hline \hline
\end{tabular}

about the electron energy distribution function and taking into account the $1+\rightarrow 2+$ electron impact ionization cross section of the incident $6 \mathrm{eV} \mathrm{Na}$ ions (calculated from the Lotz formula [21]) yields a mean-free path of $570-1570 \mathrm{~mm}$ for their in-flight ionization at the range of plasma densities listed in Table IV. The corresponding fraction of in-flight ionized $1+$ ions is $0.07-0.19$; i.e. the corresponding uncertainty is on the order of $10 \%$.

\section{DISCUSSION}

The presented study demonstrates the applicability of the $1+$ ion beam injection as diagnostics method of charge breeder ECRIS plasmas in the case of a combination of light ions $(\mathrm{Na}-\mathrm{He})$. Furthermore, the results can be compared to those reported in Ref. [6] for heavier ions and buffer gas, which allows discussion on the possible reasons for lower charge breeding efficiency often associated to light ions.

The global charge breeding efficiency of $\mathrm{Na}$ in helium plasma was measured to be approximately $20 \%-30 \%$ while the total efficiency reported for $\mathrm{Rb}$ and $\mathrm{Cs}$ in oxygen plasma is typically $50 \%-60 \%$ in the same experimental setup. Furthermore, when optimizing the charge breeder on high charge states, the fraction of the incident $\mathrm{Na}^{1+}$ ion beam propagating through the plasma is in the range from $8 \%$ to $36 \%$ (see Table I), while the corresponding values for $\mathrm{Rb}$ and $\mathrm{Cs}$ ions are in the range from $2 \%$ to $25 \%$ at equivalent microwave power level. Finally, the calculated mean-free paths of ion-ion collisions are approximately $15 \%-40 \%$ longer for $\mathrm{Na}^{1+}$ ions than for heavier alkali metal, e.g. $\mathrm{Rb}^{1+}$, ions. Altogether, this implies that the difference in charge breeding efficiencies between light and heavy ions could be partly explained by different ion-ion collision rates favoring the capture and thermalization of the heavier incident ions species. On the other hand, the contribution of in-flight ionization is more significant (up to several tens of percent) for heavier species [6]. In-flight ionization improves the capture efficiency and thermalization of the injected ions because the ion-ion collision cross section depends quadratically on the ion charge state.

The experimental results were compared to numerical simulations of the capture process carried out by the INFN ion source team $[16,17]$. The applied simulation code models the slowing down of the incident ions due to Coulomb collisions by implementing the Langevin formalism. The input parameters of the simulation include the properties of the incident ion beam, magnetic field of the charge breeder ECRIS, plasma density and ion temperature.
Ambipolar potentials and ionizations are also taken into account.

Figure 2 shows the simulated and experimental $\mathrm{Na}^{1+}$ transport efficiencies as a function of the incident ion energy for a plasma density of $4.4 \times 10^{11} \mathrm{~cm}^{-3}$ corresponding to the lower limit value estimated for $470 \mathrm{~W}$ microwave power. The simulated (total) capture efficiencies are given in parentheses associated with each data point. It was assumed for the simulation that the ion temperature in the helium plasma is $0.5 \mathrm{eV}$, the effective charge state of the plasma ions is 1.5 and the value of the Coulomb logarithm is 10 (similar to the previous section). By indicating with $V_{1+}, V_{p}$, and $V_{C B}$ the potentials of the $1+$ source, the charge breeder plasma and the charge breeder (all with respect to laboratory ground), the simulated efficiencies are a function of $\Delta V_{i n j}=V_{p}-V_{1+}$, while the experimental ones depend on $\Delta V_{\exp }=$ $V_{C B}-V_{1+}$. Taking into account that $\Delta V_{\exp }<\Delta V_{i n j}$, i.e. $V_{C B}<V_{p}$ due to the positive plasma potential, the simulated curves are shifted towards lower $\Delta V$. The shift is a measure of the plasma potential with respect to the charge breeder plasma chamber. In the present case a shift of approximately $10 \mathrm{~V}$ was observed to best reproduce the shape of the experimental $\Delta V$-curve. The value is in good agreement with the plasma potential of 14-16 V measured [22] for the PHOENIX charge breeder operated with an oxygen plasma whereas the ambipolar plasma potential of helium plasma can be expected to be slightly lower than in the case of oxygen $[23,24]$. The estimated transport efficiency of the $n+$ beam line downstream from the ECRIS is taken into account in the comparison between the simulation and the experiment.

The simulated $1+$ transport efficiencies, increasing with the incident ion energy, are in good agreement with the experimentally measured values. Also the simulated peak

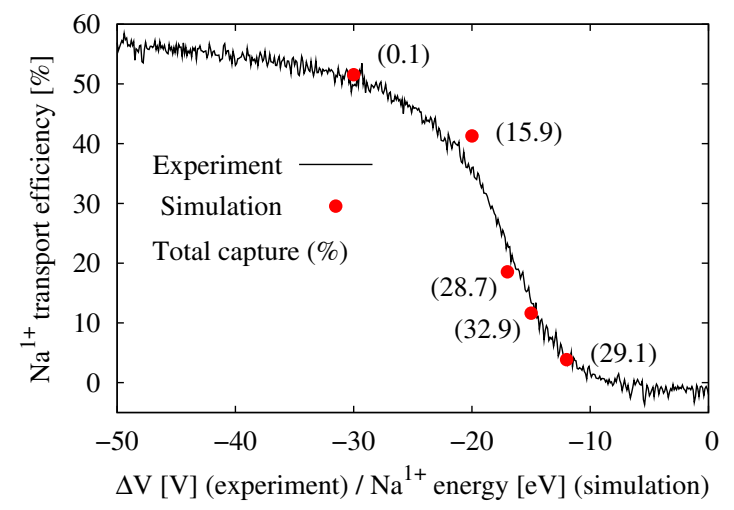

FIG. 2. Transport efficiencies of $\mathrm{Na}^{1+}$ ions through the plasma (simulation and experiment) and simulated total capture efficiency as a function of the incident ion energy. A plasma density of $4.4 \times 10^{11} \mathrm{~cm}^{-3}$ and ion temperature of $0.5 \mathrm{eV}$ were assumed for the simulation. The experimental data corresponds to $470 \mathrm{~W}$ microwave power. 
TABLE V. Transport efficiencies of incident $\mathrm{Na}^{1+}$ ions (simulation and experiment) and simulated total capture efficiency as a function of the plasma density (simulation)/microwave power (experiment).

\begin{tabular}{lcccc}
\hline \hline Simulation $\left(E_{\mathrm{Na}}=5 \mathrm{eV}\right)$ \\
\hline Plasma density $\left[\mathrm{cm}^{-3}\right]$ & $1.6 \times 10^{11}$ & $3.1 \times 10^{11}$ & $4.0 \times 10^{11}$ & $4.4 \times 10^{11}$ \\
\hline $\mathrm{Na}^{1+}$ transport [\%] & 38.0 & 20.6 & 13.9 & 11.6 \\
Total capture [\%] & 18.6 & 29.7 & 32.6 & 32.9 \\
Experiment $(\Delta V=-15 \mathrm{~V})$ & & & \\
$\mathrm{Microwave} \mathrm{Vower}[\mathrm{W}]$ & 150 & 250 & 350 & 470 \\
$\mathrm{Na}^{1+}$ transport [\%] & 46.8 & 19.0 & 17.9 & 11.9 \\
\hline \hline
\end{tabular}

of the total capture efficiency, i.e. about 30\% (as a maximum), coincides with the experimental range of $\Delta V$-values corresponding to nonzero charge breeding efficiencies of high charge states $(10 \mathrm{~V} \leq|\Delta V| \leq 20 \mathrm{~V}$, see Fig. 1). The simulated total capture efficiencies of $\mathrm{Na}$ ions in helium plasma can be compared to the simulated capture efficiencies of Rb ions in oxygen plasma, reported in Ref. [17]. The maximum capture efficiencies of $\mathrm{Na}$ and $\mathrm{Rb}$ are approximately $33 \%$ and $48 \%$, respectively. The simulated capture efficiencies are again in good agreement with the experiment indicating that the lower capture efficiency of $1+$ ions, not the subsequent ionization efficiency, is likely to be the main reason for lower breeding efficiencies of high charge state light ions $(\mathrm{Na})$ in comparison to heavier species $(\mathrm{Rb})$.

Finally, Table $\mathrm{V}$ shows the simulated and experimental $\mathrm{Na}^{1+}$ transport efficiencies as a function of the plasma density (simulation) and microwave power (experiment) at the injection energy of $5 \mathrm{eV} /$ experimental $\Delta V$ of $-15 \mathrm{~V}$, corresponding to optimized total capture efficiency. The simulated total capture efficiency is also displayed in the table. The agreement between the simulation and the experiment is reasonably good, considering that the same shift was supposed for all power levels. It is evident that the capture efficiency is improved and 1+ transport efficiency decreased with increasing plasma density.

The thermalization of the light ions could be improved by increasing the average charge state of the buffer gas plasma and/or the plasma density. This could be achieved by improving the overall performance of the ECRIS, e.g. by increasing the microwave frequency and, hence, the magnetic field strength, or by using a high-Z buffer gas. Optimizing the capture efficiency by changing the buffer gas to oxygen, for example would, however, deteriorate the breeding efficiency of high charge state $\mathrm{Na}$ ions due to unfavorable gas mixing conditions, which was confirmed during the experiments for sodium as well as reported elsewhere for potassium [9]. Altogether, this implies that the breeding efficiency of the light ions could be best improved by increasing the plasma density and optimizing the ion optics of the $1+$ ion injection [25]. The fact that the thermalized high charge state ions are highly collisional highlights the importance of the capture efficiency and optimization of the gas mixing conditions, which includes appropriately chosen buffer gas and good vacuum conditions.

\section{ACKNOWLEDGMENTS}

The authors would like to acknowledge the financial support of the ERA-NET NuPNET. This work has been supported by the Academy of Finland under the Finnish Center of Excellence Program 2012-2017 (Nuclear and Accelerator Based Physics Research at JYFL).

[1] Y. Blumenfeld, T. Nilsson, and P. Van Duppen, Facilities and methods for radioactive ion beam production, Phys. Scr. T152, 014023 (2013).

[2] R. Geller, C. Tamburella, and J. L. Belmont, The ISOLMAFIOS source (invited), Rev. Sci. Instrum. 67, 1281 (1996).

[3] R. E. Marrs and D. R. Slaughter, A high intensity electron beam ion trap for charge state boosting of radioactive ion beams, AIP Conf. Proc. 475, 322 (1999).

[4] F. Wenander, Charge breeding techniques, Nucl. Phys. A746, 40 (2004).

[5] P. Delahaye, O. Kester, C. Barton, T. Lamy, M. MarieJeanne, and F. Wenander, Evaluation of charge breeding options for EURISOL, Eur. Phys. J. A 46, 421 (2010).

[6] O. Tarvainen, T. Lamy, J. Angot, T. Thuillier, P. Delahaye, L. Maunoury, J. Choinski, L. Standylo, A. Galatà, G. Patti, and $\mathrm{H}$. Koivisto, Injected $1+$ ion beam as a diagnostics tool of charge breeder ECR ion source plasmas, Plasma Sources Sci. Technol. 24, 035014 (2015).

[7] H. Tawara and T. Kato, Total and partial ionization cross sections of atoms and ions by electron impact, At. Data Nucl. Data Tables 36, 167 (1987).

[8] T. Lamy, J. L. Bouly, J. C. Curdy, R. Geller, A. Lacoste, P. Sole, P. Sortais, T. Thuillier, J. L. Vieux-Rochaz, K. Jayamanna, M. Olivo, P. Schmor, and D. Yuan, Charge state breeding applications with the ECR PHOENIX source: From low to high current production, Rev. Sci. Instrum. 73, 717 (2002).

[9] L. Maunoury, P. Delahaye, M. Dubois, J. Angot, P. Sole, O. Bajeat, C. Barton, R. Frigot, A. Jeanne, P. Jardin, O. Kamalou, P. Lecomte, B. Osmond, G. Peschard, T. Lamy, and A. Savalle, Charge breeder for the SPIRAL1 upgrade: Preliminary results, Rev. Sci. Instrum. 87, $02 B 508$ (2016).

[10] A. G. Drentje, A. Girard, D. Hitz, and G. Melin, Role of low charge state ions in electron cyclotron resonance ion source plasmas, Rev. Sci. Instrum. 71, 623 (2000).

[11] A. A. Ivanov and K. Wiesemann, Ion confinement in electron cyclotron resonance ion sources (ECRIS): importance of nonlinear plasma-wave interaction, IEEE Trans. Plasma Sci. 33, 1743 (2005).

[12] T. A. Antaya, J. Phys. Coll. 50, 707 (1989).

[13] G. D. Shirkov, A classical model of ion confinement and losses in ECR ion sources, Plasma Sources Sci. Technol. 2, 250 (1993).

[14] H. Koivisto, O. Tarvainen, V. Toivanen, J. Komppula, R. Kronholm, T. Lamy, J. Angot, P. Delahaye, L. Maunoury, 
A. Galatà, G. Patti, L. Standylo, O. Steczkiewicz, and J. Choinski, Ionization efficiency studies with charge breeder and conventional electron cyclotron resonance ion source, Rev. Sci. Instrum. 85, $02 B 917$ (2014).

[15] J. L. Delcroix and A. Bers, Physique des Plasmas (CNRS, New York, 1994), Vol. 2, p. 357.

[16] A. Galatà et al., A new numerical description of the interaction of an ion beam with a magnetized plasma in an ECR-based charge breeding device (to be published).

[17] A. Galatà, D. Mascali, L. Neri, G. Torrisi, and L. Celona, A three-dimensional numerical modelling of the PHOENIXSPES charge breeder based on the Langevin formalism, Rev. Sci. Instrum. 87, $02 B 507$ (2016).

[18] G. Melin, A. G. Drentje, A. Girard, and D. Hitz, Ion behavior and gas mixing in electron cyclotron resonance plasmas as sources of highly charged ions, J. Appl. Phys. 86, 4772 (1999).

[19] R. Geller, T. Lamy, and P. Sortais, Charge breeding of isotope on-line-created radioactive ions using an electron cyclotron resonance ion trap, Rev. Sci. Instrum. 77, 03B107 (2006).

[20] A. Girard, D. Hitz, G. Melin, and K. Serebrennikov, Electron cyclotron resonance plasmas and electron cyclotron resonance ion sources: Physics and technology (invited), Rev. Sci. Instrum. 75, 1381 (2004).

[21] W. Lotz, Electron-impact ionization cross-sections and ionization rate coefficients for atoms and ions, Astrophys. J. Suppl. Ser. 14, 207 (1967).

[22] T. Lamy, J. Angot, M. Marie-Jeanne, J. Medard, P. Sortais, T. Thuillier, A. Galatà, H. Koivisto, and O. Tarvainen, Fine frequency tuning of the PHOENIX charge breeder used as a probe for ECRIS plasmas, in Proceedings of ECRIS2010, Grenoble, France (JACoW, 2010), WECOBK03.

[23] N. K. Bibinov, V. F. Bratsev, T. P. Kokh, P. I. Ochkur, and K. Wiesemann, Spectroscopic determination of the cold electron population in very low pressure ECR discharges in N2/He mixtures, Plasma Sources Sci. Technol. 14, 109 (2005).

[24] O. Tarvainen, P. Suominen, T. Ropponen, H. Koivisto, R. C. Vondrasek, and R. H. Scott, Plasma potential measurements with a new instrument, AIP Conf. Proc. 749, 61 (2005).

[25] R. Vondrasek, P. Delahaye, S. Kutsaev, and L. Maunoury, Improved charge breeding efficiency of light ions with an electron cyclotron resonance ion source, Rev. Sci. Instrum. 83, 113303 (2012). 